\title{
Re-distribution (condensation) of magnons in a ferromagnet under pumping
}

\author{
A.A. Zvyagin \\ B. Verkin Institute for Low Temperature Physics and Engineering of the National Academy of Sciences of Ukraine \\ 47 Lenin Ave., Kharkov 61103, Ukraine \\ E-mail: zvyagin@ilt.kharkov.ua
}

Received April 20, 2007

\begin{abstract}
Last years several experiments were performed, which studied the Bose-Einstein condensation of quasi-particles, in particular, magnons in magnetically ordered systems. Recently the Bose-Einstein condensation of magnons was observed at room temperatures in a ferromagnetic film. The theory of the condensation (re-distribution) of magnons under the conditions of pumping, which explains many features of that experiment, is presented. The use of the term «Bose-Einstein condensation of magnons» is discussed.
\end{abstract}

PACS: $03.75 . N t$ Other Bose-Einstein condensation phenomena;

75.45.+j Macroscopic quantum phenomena in magnetic systems;

76.50.+g Ferromagnetic, antiferromagnetic, and ferrimagnetic resonances; spin-wave resonance.

Keywords: condensation of magnons, parametric pumping.

The interest to the Bose-Einstein condensation (BEC) of bosonic particles and quasi-particles has been considerably increased during last years. That quantum mechanical phenomenon from the pure theoretical problem became one of the most attractive things in the modern physics, mostly due to recent experiments on dilute ultra-cold gases of atoms [1-3]. The theory of that phenomenon (BEC) for particles is well understood by now. On the other hand, the phenomenon of BEC for quasi-particles causes many discussions, and very different opinions were expressed about its nature. Among others, the BEC of magnons (magnetic quasi-particles, quanta of low-energy excitations of magnetic systems) has attracted much attention of physicists during last years [4-8]. For example, in the recent letter Demokritov et al. [9] reported the observation of BEC of magnons at room temperatures. In this study the theory, which theoretically explains many features of the experiment [9] is presented.

The observation of BEC of magnons under the conditions of the so-called parametric pumping of magnons in thin films of the yttrium-iron-garnet (YIG) was reported in [9]. Actually, the authors have studied experimentally how the spectral density of magnons $\rho(E)$, defined as $\rho(E)=D(E) n(E)$ (where $D(E)$ is the density of states of magnons, and $n(E)$ is the statistical distribution function of magnons, $E$ is the energy of magnons), is affected by the pumping by means of the external magnetic rf field.
For that purpose they used the Brillouin light scattering (BLS) spectroscopy. Under the conditions of the experiment [9] YIG can be considered as a ferromagnet (as usual for such kind of experiments, where only the lowest branch of magnetic excitations of YIG was studied). For ferromagnets it is commonly accepted that at low temperatures (and/or at their low densities) magnons satisfy the Bose-Einstein statistics, and, hence, $n(E)$ is the Bose-Einstein distribution function. In the experiment [9] the authors considered the dependence of $n, D$, and $\rho$ on the frequency $v$ rather than on the energy $E$ (using the basic quantum mechanical connection between the energy and the frequency $E=h v$, where $h \equiv 2 \pi \hbar$ is the Planck's constant).

The parametric pumping is usually performed in the parallel geometry, for which the polarization of the $\mathrm{rf}$ field is collinear to the direction of the time-independent magnetic field $H$. The Hamiltonian, which describes a ferromagnet, can be written as $\mathcal{H}=\mathcal{H}_{0}+\mathcal{H}_{1}(t)$, where

$$
\begin{aligned}
\mathcal{H}_{0}=\sum_{n, r}\left[-J \mathbf{S}_{n} \mathbf{S}_{n+r}\right. & \left.-J_{z} S_{n}^{z} S_{n+r}^{z}+B\left(S_{n}^{x} S_{n+r}^{x}-S_{n}^{y} S_{n+r}^{y}\right)\right]- \\
& -g \mu_{B} H \sum S_{n}^{z}
\end{aligned}
$$

It is supposed that the external magnetic field is directed along the $z$-axis. In this expression $S_{n}^{x, y, z}$ are operators of 
projections of the spin $S$ in the $n$th site of the crystal, $g$ is the $g$-factor, $\mu_{B}$ is the Bohr's magneton, $J>0$ is the isotropic ferromagnetic coupling, $J_{z}$ and $B$ are the coupling constants of the spin Hamiltonian of the system, related to magnetically anisotropic interactions. $J_{z}$ denotes the magnetic anisotropy of the crystal, parallel to the external magnetic field, and $B$ denotes the magnetic anisotropy, perpendicular to the direction of the field. In the standard approach $B$ is determined, e.g., by the orthorhombic magnetic anisotropy, and/or by the magnetic dipole-dipole interactions. The latter also gives a contribution to the term $J_{z}\left(J_{z}\right.$ can be caused by the uniaxial magnetic anisotropy, too) [11]. Usually, in standard magnets, including YIG, one has the condition $B<<J_{z}, g \mu_{B} H, J$. In the cubic crystal of YIG, the main contribution to $J_{z}$ and $B$ comes, probably, from the magnetic dipole-dipole coupling. The sums are performed over all sites, and $r=0,1,2, \ldots$ determines radii of spin-spin interactions. For example, for possible (in principle) terms, describing a single-ion magnetic anisotropy, one has to put $r=0$. Obviously, the magnetically isotropic spin-spin interaction $J$ does not change the directions of spins of the ferromagnet in the equilibrium. However, the isotropic spin-spin ferromagnetic coupling is responsible for the fact, that all spins of the ferromagnet are parallel to each other in the ground state. For the description of the low-temperature thermodynamic characteristics of the ferromagnet the spin-wave approximation is often used [11]. In that approximation one replaces operators of quantum spins by classical vectors, and looks for a steady-state (equilibrium) configuration of those vectors. That steady state is associated with the ground state of the original quantum spin system. Then small (inhomogeneous) deviations of classical vectors from that steadystate configuration are called spin waves, or, in the quantized form, magnons [11]. The Hamiltonian Eq. (1) in that approximation can be written as

$$
\mathcal{H}_{0} \approx E_{0}+\sum_{\mathbf{k}} E_{\mathbf{k}} \hat{n}_{\mathbf{k}}
$$

where $E_{0}$ is determined by the energy of the lowest energy equilibrium configuration, $E_{\mathbf{k}}\left(E_{\mathbf{k}}=E_{-\mathbf{k}}\right.$ for the considered case) and $\hat{n}_{\mathbf{k}}$ are the energy and the operator of the number of magnons with quasi-momenta $\mathbf{k} . E_{\mathbf{k}}$ depends on $J, J_{z}, B$, and $H$ in the standard way [11]. For the considered ferromagnet the minimal energy of magnons is determined by the value of the spin gap, which does not depend on the isotropic ferromagnetic interaction $J$. When calculating thermodynamic characteristics of a ferromagnet it is often useful to replace the summation over quasi-momenta by the integration over energies $\Sigma_{\mathbf{k}} \rightarrow V_{0} \int D(E) d E$, where $D(E)$ is the density of states of magnons, and $V_{0}$ is the phase volume. That is why, for example, the low-temperature energy of the ferromagnet in that approximation is equal to

$$
E_{l t} \approx E_{0}+V_{0} \int E n(E) D(E) d E \equiv E_{0}+V_{0} \int \rho(E) E d E,
$$

where $n(E)$ is the Bose-Einstein distribution function. To remind, in this work, following the letter [9], we use $E=h v$, i.e. the integration over energies $E$ are replaced by integration over frequencies $v$. It is important to point out that because of the spin gap, the integration over $v$ (or over $E$ ) is, in fact, performed over the interval $v \geq v_{m}$ (as well as $v \leq v_{\max }$ ), where $h v_{m}$ is determined by the spin gap of $E_{\mathbf{k}}\left(E_{\mathbf{k}}=\sqrt{\left(h v_{m}\right)^{2}+f(\mathbf{k})}\right.$ in our model, where $\left.\lim _{\mathbf{k} \rightarrow 0} f(\mathbf{k})=0\right)$, and $v_{\max }$ is related to the maximal energy of magnons. This property follows from the density of states of magnons of the considered ferromagnet $D(v)$. The term of the Hamiltonian, describing the effect of the external $\mathrm{rf}$ magnetic field, is given by the expression

$$
\mathcal{H}_{1}(t)=-g \mu_{B} h_{0} \cos \left(2 \pi v_{p} t\right) \sum_{n} S_{n}^{z}
$$

Here $h_{0}$ and $v_{p}$ are the magnitude and the frequency of the rf magnetic field, respectively. In this geometry in the magnon approximation, the resonance takes place if the frequency of the rf magnetic field is equal to the energy of two magnons with opposite directions of quasi-momenta of magnons, $E_{\mathbf{k}}$ and $E_{-\mathbf{k}}$, i.e. at $h v_{p}=E_{\mathbf{k}}+E_{-\mathbf{k}}$ ( $=2 E_{\mathbf{k}} \equiv 2 h v$ in the considered case).

Several features of the behavior of the BLS spectral density of magnons were observed in the experiment [9]. Namely, when the power $P$ of the rf pumping field was weak enough, the observed BLS spectral function was fitted with the help of the same density of states of magnons and the same Bose-Einstein distribution function of magnons, which were observed in YIG without pumping. The intensities of the observed BLS spectra of magnons (related to the spectral density of magnons) increased when the power of the rf magnetic field became larger. The intensity of the BLS spectra increased also with the time of the action of pumping, until saturation at approximately 400-500 ns. Before saturation the BLS spectra were also possible to fit with the help of the above mentioned procedure. However, in the saturation regime, it was already impossible to fit the spectra with the help of the standard Bose-Einstein distribution function. Demokritov et al. pointed out that to reach the agreement with the experiment it was necessary to add (by hand, without derivation) to the distribution function of magnons $n(v)$ a singularity peak $\propto \delta\left(v-v_{m}\right)$, where $v_{m}$ was the frequency of the maximum of the intensity in the observed BLS spectra of magnons (which corresponds to the minimal energy of magnons $E_{\mathbf{k}}$ ). To explain the appearance of the singularity peak the authors of Ref. [9] supposed that in the steady-state regime, for $t>400-500 \mathrm{~ns}$, BEC of magnons took place (at room temperature, at which the exper- 
iment was performed). They concluded that the re-distribution of magnons under pumping was related to BEC.

Here we propose the theory, which explains many features of the experiment of Demokritov et al. For that purpose we use the approach of the paper [10]. It was shown in [10] that under the condition of the parametric pumping of magnons in a ferromagnet the steady-state configuration of spins (or classical vectors, see above) in the effective Hamiltonian, which determines the time evolution of the system under parametric pumping, is changed in resonance. It is very similar to the procedure, outlined above for the description of low-temperature thermodynamic characteristics of a ferromagnet, but with the application to the effective Hamiltonian, which describes the low-energy dynamics of the spin system under pumping. In this work we generalize the approach of [10]. It is easy to show that out of resonance each spin (or a classical vector) of that effective Hamiltonian at equilibrium has the projection to the axis, parallel to the direction of the external magnetic field $S_{0}^{z}=S$ (other projections are equal to $\left.S_{0}^{x} \approx S_{0}^{y}=(1 / 2) \sqrt{S^{2}-\left(S_{0}^{z}\right)^{2}}\right)$. On the other hand, in resonance the configuration at equilibrium of spins (vectors) in the effective Hamiltonian is such that the $z$-projection of the effective spin (classical vector) becomes equal to

$$
S_{0}^{z}=\frac{2 g \mu_{B} H-h v_{p}}{4\left(B\left(g \mu_{B} h_{0} / h v_{p}\right)-J_{z}\right)},
$$

where the small parameter $g \mu_{B} h_{0} / h v_{p}<<1$ was used. One can see that at resonance (when the frequency of the rf field is close to the value $v_{p}=E_{\mathbf{k}} / \pi \hbar$ ), the effective position of equilibrium of spins can be changed dramatically.

All characteristics of any quantum system in mixed states depend on the density matrix $\rho_{d}$. The time evolution of the latter can be determined by the solution of the Liouville-like equation [10]

$$
i \hbar\left(\frac{\partial \rho_{d}}{\partial t}\right)=\left[\mathcal{H}, \rho_{d}\right]-i \hbar \gamma\left(\rho_{d}-\rho_{0}\right) .
$$

Here [.,.] denotes the commutator, $\gamma$ is the relaxation constant (the relaxation is introduced in the simplest form), and $\rho_{0}$ is the density matrix in the equilibrium. It is worthwhile to suppose, following the paper [10], that the density matrix in equilibrium is determined by the Hamiltonian of the system without pumping $\mathcal{H}_{0} \equiv \mathcal{H}\left(h_{0}=0\right)$,

$$
\rho_{0}=\frac{\exp \left(-\mathcal{H}_{0} / k_{B} T\right)}{\operatorname{Tr}\left(\exp \left(-\mathcal{H}_{0} / k_{B} T\right)\right)},
$$

where $k_{B}$ is the Boltzmann's constant, and $T$ is the temperature. Such an assumption is justified, because usually the magnitude of the pumping field $h_{0}$ is much smaller than the value of the stationary field $H$. At low temperatures we can replace $\mathcal{H}_{0}$ in Eq. (7) by the approximate Hamiltonian of the free gas of magnons $\mathcal{H}_{0} \approx E_{0}+\sum_{\mathbf{k}} E_{\mathbf{k}} \hat{n}_{\mathbf{k}}$, see the discussion above. On the other hand, the dynamics of the spin system under pumping is determined by the effective Hamiltonian, mentioned above. We look for the solution of Eq. (6) using the standard set of unitary transformations [10], and the resonance approximation.

The solution of the Liouville-like equation manifests two different regimes, cf. [10]. In the first (dynamical) regime, at $t<<\gamma^{-1}$, all characteristics of the system oscillate with two frequencies: the frequency of the pumping field $v_{p}$, and the small frequency of Rabi-like oscillations, which value is determined in resonance by the magnitude of the rf field $h_{0}$. On the other hand, in the second (steady-state) regime, at $t>>\gamma^{-1}$, only oscillations with $v_{p}$ survive. We are interested in the contributions to the spectral density of magnons, which are proportional to $\sin \left(2 \pi v_{p} t\right)$, because the main contribution to the density comes from the term

$$
\overline{\left(\frac{\partial \mathcal{H}_{1}}{\partial t}\right)}=2 \pi v_{p} g \mu_{B} h_{0} \overline{\sin \left(2 \pi v_{p} t\right) \sum_{n}\left\langle S_{n}^{z}\right\rangle},
$$

where the line denotes the time averaging

$$
\overline{f(t)}=\lim _{A \rightarrow \infty} A^{-1} \int_{0}^{A} f(t) d t,
$$

and brackets denote the thermal averaging. Following the approach of the paper [10] and using the small parameter $g \mu_{B} h_{0} / h v_{p}<<1$, we find in the steady-state regime, at $t>>\gamma^{-1}$, that the (small) addition to the spectral function of magnons $\delta \rho(v)$, caused by the pumping, is proportional to

$$
\delta \rho(v) \sim D(v) n_{0}(v) \frac{g \mu_{B} h_{0}}{h v_{p}} \frac{2\left(B S_{0}^{x}\right)^{2}}{g \mu_{B} H} \frac{\hbar \gamma}{(\hbar \gamma)^{2}+\varepsilon^{2}},
$$

where

$$
\varepsilon^{2}=\left[g \mu_{B} H+2 J_{z} S_{0}^{z} Z-\left(h v_{p} / 2\right)\right]^{2}+\left(\frac{g \mu_{B} h_{0}}{h v_{p}} 2 B S_{0}^{x}\right)^{2},
$$

$Z$ is the coordination number, and $n_{0}(v)$ is the Bose-Einstein distribution function of magnons without pumping, which is determined by the Hamiltonian $\mathcal{H}_{0}$ in the spinwave approximation. The resonance in the parallel geometry of pumping takes place at $v_{p}=2 v$. To show that, we can write down the Hamiltonian of the system in the quasi-classical (magnon) approximation:

$$
\begin{gathered}
\mathcal{H}=E_{0}^{\prime}+\sum_{\mathbf{k}} E_{\mathbf{k}} \hat{n}_{\mathbf{k}}+ \\
+\frac{g \mu_{B} h_{0} B S_{0}^{x}}{E_{\mathbf{k}}} \cos \left(2 \pi v_{p} t\right) \sum_{\mathbf{k}}\left(b_{-\mathbf{k}}^{\dagger} b_{\mathbf{k}}^{\dagger}+\text { H.c. }\right)+\ldots
\end{gathered}
$$


Here $E_{0}^{\prime}$ does not depend on operators, $b_{\mathbf{k}}^{\dagger}$ are the creation operators of magnons with the quasi-momentak $\left(\hat{n}_{\mathbf{k}} \equiv b_{\mathbf{k}}^{\dagger} b_{\mathbf{k}}\right.$ ), and H.c. denote, as usually, Hermitian conjugated terms. From this formula one immediately sees that the resonance terms, i.e. those, which give the nonzero contribution to the linear in $g \mu_{B} h_{0} / E_{\mathbf{k}}$ response of the spin system to the rf field, are those, for which $E_{\mathbf{k}}=$ $=h v_{p} / 2$, or $h v=h v_{p} / 2$. That procedure for resonance terms is similar to the procedure for the obtaining the solution of Eq. (6). First, using the unitary transformation

$$
U_{1}=\exp \left(i \frac{g \mu_{B} h_{0}}{h v_{p}} \sin \left(2 \pi v_{p} t\right) \sum_{n} S_{n}^{z}\right),
$$

the time dependence is transferred to the non-diagonal terms of the Hamiltonian $\mathcal{H}$. Then, we used the small parameter $g \mu_{B} h_{0} / h \nu_{p}$, and the resonance approximation. The latter means that we drop all the terms in the obtained effective Hamiltonian, which determines the spin dynamics, except of those, which give a nonzero contribution in the linear in $g \mu_{B} h_{0} / h v_{p}$ response of the system. Other terms, which depend on $g \mu_{B} h_{0}$ (the resonance ones) are taken into account exactly. Then, the explicit dependence on time is removed from the effective Hamiltonian, using the unitary transformation

$$
U_{2}=\exp \left(i \pi v_{p} \sum_{n} S_{n}^{z}\right) .
$$

In the obtained Hamiltonian, which determines the spin dynamics of the system, we use the quasi-classical approximation, taking into account the shift of the steadystate configuration of spins in resonance. And, finally, we use the unitary transformation

$$
U_{3}=\exp (-i \tilde{\mathcal{H}} / t)
$$

where $\widetilde{\mathcal{H}}$ is the obtained effective Hamiltonian, which determines the spin dynamics. The time dependence for $\rho_{d}(t)$ is obtained by using the consecutive combination of the above described procedures. The average value of the operator $\hat{n}$ is obtained after averaging it with $\rho_{d}(t, T)$, and the temperature dependence in this approach is determined by Eq. (7). Out of resonance, where $v \neq v_{p} / 2$, the addition to the spectral function, caused by the pumping, is approximately zero. In resonance, one may substitute $v_{p} / 2=v$ in the first term of Eq. (11), see above. Namely that substitution (justified at resonance) produces the re-distribution of the spectral density of magnons in Eq. (10). This is why, the addition $\delta \rho(v)$ in the steady-state regime in resonance is approximately proportional to $\delta\left(v-v_{m}\right)$ (notice that for our system in the used approximation one has $h v_{m} \approx$ $\left.\approx g \mu_{B} H+2 J_{z} S_{0}^{z} Z+O(B)\right)$. Here we used the formula $\lim _{\hbar \gamma \rightarrow 0} \hbar \gamma /\left[(\hbar \gamma)^{2}+x^{2}\right] \propto \delta(x)$. Hence, the pumping causes the coherent condensation of magnons to the state with the minimal energy $h v_{m}$. One can see that even if the half-frequency of the pumping field $v_{p} / 2$ is larger than $v_{m}$, but it is in resonance with some of higher-energy magnons (i.e. $2 v_{m} \leq v_{p} \leq 2 v_{\max }$ ), our theory predicts the re-distribution of the magnon spectral density to the one with the peak $\propto \delta\left(v-v_{m}\right)$ near the minimal energy of magnons. That re-distribution means, that when the photon of the pumping field is in resonance with spins of the system, those spins dynamically change their effective equilibrium configuration in such a way, that some part of small deviations of that dynamical equilibrium (magnons) becomes re-distributed at the lowest edge of the magnon spectrum. The physical reason for the re-distribution to the minimal energy of magnons is due to the fact that in our approximation $\left(B<<g \mu_{B} H, J_{z}, J\right)$ the k-dependent dispersion of the energy of magnons is related to the isotropic ferromagnetic interaction, which operator commutes with the $z$-projection of the total spin of the system (to which the rf pumping field is connected). It also turns out, that the addition $\delta \rho(v)$ is increased with the growth of the pumping power $P$ (because $P$ is proportional to $h_{0}^{2}$ ).

On the other hand, we calculated that for $t<<\gamma^{-1}$, in the dynamical regime, the small contribution to the spectral density of magnons, caused by the pumping, is proportional (at small $t$, at which one can use the approximation $\sin (\varepsilon t / \hbar) \approx \varepsilon t / \hbar)$ to

$$
\delta \rho(v) \sim D(v) n_{0}(v) \frac{g \mu_{B} h_{0}}{h v_{p}} \frac{2\left(B S_{0}^{x}\right)^{2}}{g \mu_{B} H} \frac{t}{\hbar} .
$$

Obviously, in that regime the spectral function of magnons is increased with time (as well as with the power of the pumping field $P$ ). However, in this regime there is no $\delta$-function contribution to the spectral function, and the latter (even in resonance) is determined by the distribution function of magnons without pumping. Such a behavior is also similar to what was observed in [9].

One can see that the present theory describes many features of the experiment of Demokritov et al. [9]. First, in the dynamical regime, the spectral function of magnons is increased with time, reaching the steady-state regime. There the pumping renormalizes the distribution of magnons in such a way, that in resonance some part of magnons is in the coherent state with the lowest possible energy (approximately with the energy of the spin gap). Demokritov et al. called such a process BEC of magnons. However, one can express doubts about the correctness of the use of such a term for magnons. It is important to point out that BEC determines the ground state of bosonic particles. The situation is drastically different for magnons. It is well known that for many magnetically ordered systems (and, moreover, for magnetic systems with the spin frustration) in most of cases the ground state cannot be found theoretically exactly [11]. Usually (see above) the quasi-classical approximation is used, when one replaces the operators of quantum spins by their classical counter- 
parts (vectors), then the steady-state configuration of those classical vectors is associated with the ground state of quantum spins. Small deviations from that steady-state configuration are called spin waves (or, in the quantized form, magnons). However, BEC for magnons implies the renormalization of the ground state: All magnons at $T<T_{B E C}$ (and, thus, at $T=0$ also) are re-distributed to the BEC state. Then, one faces with the paradoxical situation: The ground state of the spin system was not determined exactly, but the use of such a term, BEC of magnons, implies the renormalization of the (unknown!) ground state of the spin system. Hence, such a use is under question. One can also put similar questions to the discussion of the results [4-7]. These our doubts correlate with the recent opinion of Snoke [8]. Notice, however, that, on the other hand, according to the present theory, it seems to be clear that magnons in the experiment of Demokritov et al. were re-distributed by the parametric pumping to the dynamically induced coherent quantum state, cf. [8]. Despite the question, whether the Bose-Einstein condensation is the right term to call the observed phenomena, coherent quantum states of magnons, either near phase transitions [4-7], or induced by the parametric pumping [9], are among the most interesting phenomena of the modern condensed matter physics.

The author dedicates this paper to the memory of his father, Anatolii Illarionovich Zvyagin.
1. M.H. Anderson, J.R. Ensher, M.R. Matthews, C.E. Wieman, and E.A. Cornell, Science 269, 198 (1995).

2. I. Bloch, T.W. Hänsch, and T. Esslinger, Nature 403, 166 (2000).

3. C.I. Hancox, S.C. Doret, M.T. Hummon, L.J. Luo, and J.M. Doyle, Nature 431, 281 (2004).

4. R. Coldea, D.A. Tennant, K. Habicht, P. Smeibidl, C. Walters, and Z. Tylczynski, Phys. Rev. Lett. 88, 137203 (2002).

5. Ch. Rüegg, N. Cavadini, A. Furrer, H.U. Güdel, K. Krämer, H. Mutka, A. Wildes, K. Habicht, and P. Worderwisch, $\mathrm{Na}$ ture 423, 62 (2003).

6. E. Della-Torre, L.H. Bennett, and R.E. Watson, Phys. Rev. Lett. 94, 147210 (2005).

7. T. Radu, H. Wilhelm, V. Yushankhai, D. Kovrizhin, R. Coldea, Z. Tylczynski, T. Lühmann, and F. Steglich, Phys. Rev. Lett. 95, 127202 (2005).

8. D. Snoke, Nature 443, 403 (2006).

9. S.O. Demokritov, V.E. Demidov, O. Dzyapko, G.A. Melkov, A.A. Serga, B. Hillebrands, and A.N. Slavin, Nature 443, 430 (2006).

10. A.A. Zvyagin, and V.M. Tsukernik, Fiz. Nizk. Temp. 11, 88 (1985) [Sov. J. Low Temp. Phys. 11, 47 (1985)].

11. See, e.g., R.M. White, Quantum Theory of Magnetism. Springer-Verlag, Berlin (1983). 